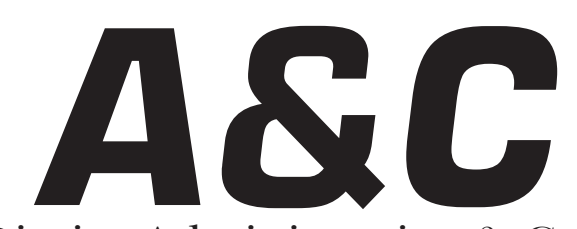

Revista de Direito Administrativo \& Constitucional

Editora Fórum

ISSN 1516-3210

\begin{tabular}{|l|l|l|l|l|l|}
\hline A\&C R. de Dir. Administrativo \& Constitucional & Belo Horizonte & ano 7 & n. 30 & p. 1-254 & out./dez. 2007 \\
\hline
\end{tabular}




\section{A\&C REVISTA DE DIREITO ADMINISTRATIVO \& CONSTITUCIONAL}

IPDA

Instituto Paranaense

de Direito Administrativo

Direção Geral

Romeu Felipe Bacellar Filho

Direção Editorial

Paulo Roberto Ferreira Motta

Direção Executiva

Emerson Gabardo

Conselho de Redação

Edgar Chiuratto Guimarães

Adriana da Costa Ricardo Schier

Célio Heitor Guimarães

\section{Conselho Editorial}

Jorge Luís Salomoni - in memoriam (Argentina)

José Carlos Abraão (Brasil)

José Eduardo Martins Cardoso (Brasil)

José Luís Said (Argentina)

José Mario Serrate Paz (Uruguai)

Juan Pablo Cajarville Peruffo (Uruguai)

Juarez Freitas (Brasil)

Julio Rodolfo Comadira - in memoriam (Argentina)

Luís Enrique Chase Plate (Paraguai)

Lúcia Valle Figueiredo (Brasil)

Manoel de Oliveira Franco Sobrinho -

in memoriam (Brasil)

in memoriam (Brasil)

Marçal Justen Filho (Brasil)

Marcelo Figueiredo (Brasil)
Márcio Cammarosano (Brasil)
Maria Cristina Cesar de Oliveira (Brasil)

Nelson Figueiredo (Brasil)

Odilon Borges Junior (Brasil)

Pascual Caiella (Argentina)

Paulo Eduardo Garrido Modesto (Brasil)

Paulo Henrique Blasi (Brasil)

Paulo Neves de Carvalho - in memoriam

(Brasil)

Paulo Ricardo Schier (Brasil)

Pedro Paulo de Almeida Dutra (Brasil)

Regina Maria Macedo Nery Ferrari (Brasil)

Rogério Gesta Leal (Brasil)

Rolando Pantoja Bauzá (Chile)

Sérgio Ferraz (Brasil)

Valmir Pontes Filho (Brasil)

Yara Stropa (Brasil)

Weida Zancaner (Brasil)

A246 A\&C Revista de Direito Administrativo \& Constitucional. ano 3, n. 11, jan./mar. 2003. Belo Horizonte: Fórum, 2003.

Trimestral

ano 1, n. 1, 1999 até ano 2, n.10, 2002 publicada pela

Editora Juruá em Curitiba

ISSN 1516-3210

1. Direito Administrativo. 2. Direito Constitucional.

I. Fórum.

CDD: 342 CDU: 33.342

(C) Editora Fórum Ltda. 2007

Todos os direitos reservados. É proibida a reprodução total ou parcial, de qualquer forma ou por qualquer meio eletrônico ou mecânico, inclusive através de processos xerográficos, de fotocópias ou de gravação, sem permissão por escrito do possuidor dos direitos de cópias (Lei n 9.610, de 19.02.1998).

Editora Fórum Ltda

Av. Afonso Pena, 2770 - 15\%16 andar - Funcionários

CEP 30130-007 - Belo Horizonte/MG - Brasil

Tel.: 08007043737

Internet: www.editoraforum.com.br

e-mail: editoraforum@editoraforum.com.br
Editor responsável: Luís Cláudio Rodrigues Ferreira Coordenação editorial: Olga M. A. Sousa

Pesquisa jurídica: Fátima Ribeiro - OAB/MG 74868

Revisora: Bárbara Christiane

Projeto gráfico: Luis Alberto Pimenta

Diagramação: Marcelo Belico

Bibliotecária: Alessandra Rodrigues da Silva -

CRB 2459 - 6 ${ }^{a}$ região

Os conceitos e opiniões expressas nos trabalhos assinados são de responsabilidade exclusiva de seus autores.

Impressa no Brasil / Printed in Brazil

Distribuída em todo o Território Nacional 


\title{
¿Eficiencia económica del Estado como substituto a la ética pública convencio- nal? Desafíos actuales del Estado para la consecución de la eficiencia económica. Su aplicación en el sector de la seguri- dad marítima
}

\author{
María Remedios Zamora Roselló \\ Becaria de Investigación del Ministerio de Educación y Ciencia. Área de Derecho Administrativo, \\ Universidad de Málaga (España).
}

\begin{abstract}
Palabras clave: Eficiencia económica del Estado. Modelos de Estado. Sector de la seguridad marítima.

Índice: $1 \mathrm{El}$ carácter obsoleto de los modelos tradicionales. Deficiencias y críticas - 2 Desafíos actuales del Estado para la consecución de la eficiencia económica. Su aplicación en el sector de la seguridad marítima - 3 Reflexiones finales - Bibliografía
\end{abstract}

\section{El carácter obsoleto de los modelos tradicionales. Deficiencias y críticas}

Los modelos de Estado tradicionales no responden a las necesidades de las sociedades actuales, ya que tienen como referencia realidades sociales superadas. Las últimas etapas del Estado del Bienestar se han caracterizado por una desproporción en el gasto público, que no iba acompañada de una mejora en la calidad de los servicios. Es evidente que el Estado del Bienestar también ha llevado aparejado la obtención de un conjunto de logros sociales; en base a los principios del Bienestar, por vez primera el Estado asumió compromisos en las áreas de educación, sanidad o desempleo, entre otras.

Sin embargo, el intervencionismo estatal también se acompañó de un aumento significativo en la burocracia, que no se tradujo en una mayor eficiencia; si no que ayudó a profundizar en el distanciamiento entre el Estado y la sociedad. Por tanto, no se alcanzó la aspiración última de esta categoría de Estado, ya que no lograba satisfacer las necesidades de

A\&C R. de Dir. Administrativo \& Constitucional, Belo Horizonte, ano 7, n. 30, p. 131-139, out./dez. 2007 
la sociedad. En definitiva, el Estado entre cuyos fines básicos se encuentra la consecución del bienestar por parte de sus ciudadanos, fracasó. Como complemento a estas desviaciones, los ciudadanos y el sector privado han identificado al sector público con sus actuaciones de carácter paternalista, y esperan que solvente todas sus necesidades, sin contraprestación alguna, en detrimento de la autorresponsabilidad social y empresarial.

Se hace necesario plantear qué debemos entender por eficiencia económica del Estado. En principio parecen dos conceptos contrapuestos, eficiencia económica y Estado, es un binomio con escasa tradición, pero que debe ser exigido a los Gobiernos. Tradicionalmente la eficiencia económica sólo podía ser exigida al sector privado, el ámbito público se encontraba más allá de estos límites, ya que no necesitaba controlar la adecuación entre gastos e ingresos. Sin embargo este tópico ha sido eliminado, uno de los ejemplos más significativos se encuentra en la propia Unión Europea, cuyo proceso para la consecución de la unión monetaria se ha encontrado determinado por la exigencia de estabilidad económica, con claras exigencias para los Estados en lo que respecta al control del gasto público y de la inflación.

Debemos cuestionarnos si este cambio de mentalidad significa que el Estado debe ocupar la categoría de una multinacional subsidiaria, regido por las reglas del mercado, y determinado por su actuación controlada en aquellos ámbitos en que ni la sociedad civil ni la iniciativa privada operen, aún conociendo su carácter imprescindible. A pesar de la voluntad de algunos sectores por equiparar el régimen de actuación del sector público y el privado, existen dos elementos claves que los distinguen: la competencia y el enriquecimiento. Algunos Estados han intentado introducir estos elementos en la actuación del sector público, a través de medidas económicas de incentivos a su personal, y también mediante el establecimiento de concursos públicos en los que las empresas compiten para la obtención de una concesión de servicio público. Sin embargo, estos intentos no han colmado las expectativas que despertaron, y han puesto de manifiesto que la metodología del Estado difiere del sector privado, ya que sus fines y funciones no pueden ser equiparados.

Este panorama se completa con el distanciamiento social de las instituciones públicas, el ciudadano no se identifica con los fines del sector público, ya que percibe que el Estado se ha distanciado de sus intereses, 
y su actuación es ineficaz. La excesiva burocracia y la corrupción minan la confianza de la sociedad en la Administración, debilitando el vínculo de relación entre las mismas. En los Estados miembros de la Unión Europea existe una marcada tendencia a la desafección de la sociedad con el Estado, que se manifesta en el distanciamiento entre el ciudadano y los partidos políticos. En el caso de los Estados comunitarios la situación se agrava por un dato más, existen dos versiones del sector público: las instituciones comunitarias y las instituciones nacionales. El ciudadano tiene como primera referencia las iniciativas públicas nacionales, y en un nivel secundario las comunitarias, percibiendo un mayor grado de eficiencia en las medidas adoptadas por las instituciones comunitarias, frente a las decisiones públicas nacionales.

\section{Desafíos actuales del Estado para la consecución de la eficiencia económica. Su aplicación en el sector de la seguridad marítima}

La perspectiva ambiental en el medio marino representa un ámbito clave que permite desarrollar una nueva dimensión de la eficiencia económica pública, alejada de los postulados tradicionales. En primer lugar, la configuración del medio ambiente como un campo para la implantación de las políticas públicas, tiene su origen en el último tercio del siglo pasado, por lo que es una materia que aún no se ha imbuido por completo de los principios tradicionales del sector público, ya que no se encuentra asentada. Este hecho se agrava en lo que respecta a la protección del medio ambiente marino, un sector relegado a un segundo término, y que sólo en los últimos años, como consecuencia de las catástrofes ambientales que han asolado mares y océanos, parece que comienza a despertar el interés de la sociedad.

Otro dato que permite otorgar una posición destacada a esta materia es su configuración más allá de las fronteras nacionales y regionales. Los caracteres del medio marino convierten su protección y conservación en una tarea que implica a toda la comunidad internacional. Por tanto, podemos afirmar que es el instrumento primigenio de un mundo globalizado, ya que no conoce de fronteras nacionales ni regionales, y su gobierno exige de medidas adoptadas en foros internacionales. Este carácter extraterritorial, favorece la integración de uno de los elementos claves en la configuración de un nuevo modelo de gestión pública: la consideración del Estado como

A\&C R. de Dir. Administrativo \& Constitucional, Belo Horizonte, ano 7, n. 30, p. 131-139, out./dez. 2007 
sujeto de una comunidad más amplia, en la que interrelacionan todos sus miembros. El Estado deja de ser considerado como elemento último e integrador, para convertirse en una pieza más del entramado de relaciones entre sujetos públicos y privados y los movimientos sociales.

La integración del ciudadano, y las organizaciones y movimientos sociales, frente a la neutralización de los mismos por parte de un Estado excesivamente intervencionista, representa uno de los desafíos más destacados a los que se enfrenta el nuevo modelo público. La protección del medio ambiente marino comenzó a formar parte de la agenda de los Estados y los organismos internacionales gracias a la presión ciudadana. Asociaciones ecologistas, ciudadanos particulares, medios de comunicación y ONGs han exigido la creación de políticas públicas para la protección y conservación del medio marino. Las reticencias estatales en la creación de una estructura para la protección y conservación de la riqueza marina, se desarrollaron paralelamente al nacimiento de destacados grupos de presión que actuaban de forma privada para la protección del patrimonio marino. Este esquema de actuación muestra que, a diferencia de las teorías que postulaban la desaparición de los movimientos sociales, éstos se han transformado, para albergar en su seno a los nuevos intereses de una sociedad evolucionada.

La industria marítima ha impuesto sus reglas durante siglos en el medio marino. La actuación de las organizaciones internacionales, y de los Estados, comenzó como una tímida respuesta a las necesidades que quedaban sin cubrir en un sector cada vez más en auge. Sin embargo, los costes ambientales de la explotación indiscriminada de los recursos marítimos, no sólo como vías de comunicación, si no también como sumidero indiscriminado, y origen de recursos alimenticios, minerales, energéticos y medicinales, no han sido asumidos. El sector público se ha visto obligado, a incorporar sistemas para la supervisión y el control de estas actividades; no obstante, los medios públicos resultan insuficientes, y los Estados no tienen la capacidad suficiente para incorporar el adecuado control. Entre los medios de control de la seguridad marítima se encuentran las inspecciones que se realizan en los Estados rectores de los puertos que reciben a los buques; las autoridades portuarias se encuentran desbordadas, y no pueden inspeccionar a todos los buques que arriban a sus puertos, por lo que se ven obligadas a realizar inspecciones en base a una serie de parámentros 
que miden el riesgo para la seguridad marítima que representa el buque.

Por tanto, en el ámbito de la seguridad marítima, nos encontramos con un sector cuyas irregularidades, irrelevantes para el ámbito privado, representan un grave riesgo para la conservación y protección del medio marino, y la salud y bienestar de los ciudadanos. En este supuesto, no puede ser vinculado el bienestar a un limitado número de habitantes de cualquier Estado, o área geográfica, si no que el equilibrio ambiental de todo el planeta se encuentra comprometido. La sociedad se ha movilizado, exige actuaciones y un endurecimiento de las exigencias a todos los armadores; el sector público comienza a actuar, pero sus medios son limitados.

$\mathrm{Ni}$ siquiera cabe plantearse en esta fase la eficiencia económica; incluso podría ser argumentado que la protección ambiental y la salud de los ciudadanos es competencia exclusiva del Estado, como garante último de su bienestar, que tendrá que asumir este coste. Si continuamos con esta línea argumental, y a la vista de las exigencias de control del gasto público y limitaciones a que se encuentra sometida la Administración Pública, debería de mantenerse esta línea de actuación de primer grado, a pesar de su mínima eficacia. Igualmente ineficaz resultaría la intervención en este sector del Estado, limitando la capacidad de actuación de la industria marítima; actuación contraria a los principios básicos del régimen de libre mercado, imperante en los Estados de la Unión Europea, así como en la mayoría de Estados.

La presión de la sociedad civil no sólo se ha traducido en la adopción de medidas por el sector público. El sector marítimo también se ha visto condicionado a cambiar su modelo de actuación. El consumidor selecciona los productos que adquiere o los servicios que contrata en función de múltiples factores, entre los que en la actualidad, ya se incluye la protección ambiental. Las etiquetas ecológicas, son una de las manifestaciones más destacadas de este fenómeno; las empresas navieras obstentan en su publicidad los certificados ambientales, de organismos privados y públicos, que constatan su respeto con el medio ambiente.

Cómo lograr la eficiencia económica en un modelo de actuación como el que estamos analizando para la mejora y protección de la seguridad marítima, es un interrogante que parece utópico, especialmente en un sector como el ambiental, que precisamente por su carácter de universal, parece que se encuentra tan alejado de cualquier interés privado o público.

A\&C R. de Dir. Administrativo \& Constitucional, Belo Horizonte, ano 7, n. 30, p. 131-139, out./dez. 2007 
No obstante, y a pesar de estas dificultades iniciales, son numerosas las iniciativas que han sido adoptadas para reducir el gasto público y si bien no rentabilizar, al menos maximizar los beneficios que puedan ser obtenidos con las partidas presupuestarias públicas; iniciativas innovadoras que han permitido captar la atención del sector privado de la propia sociedad civil.

En primer lugar, y como dato más significativo hay que destacar que el sector público ha comenzado a compartir los costes que debe asumir sobre seguridad marítima con el sector privado. Tradicionalmente, y como consecuencia de la ausencia de valoración del medio marino, los sujetos privados que participaban en su explotación no asumían los costes de su protección y conservación. Además de los cambios operados por este sector como consecuencia de la presión de los consumidores "con conciencia ambiental", el sector público también ha comenzado a instaurar medidas para la asunción de gastos por parte de quién más beneficio obtiene del medio marino. Son destacados los ejemplos de los Estados que comienzan a endurecer sus ordenamientos a través de exigencias económicas que permitan reducir los gastos asumidos por las arcas públicas.

En este sentido, podemos destacar medidas de diversa índole, no sólo de carácter reparador, como la asunción de los costes que ocasionan la reparación del entorno natural, si no también preventivo, como la constitución de garantías financieras previas a la entrada de un buque necesitado de asistencia en un lugar de refugio. Asimismo, destacan iniciativas como la creación de certificados de calidad estatales, que permiten al buque eludir inspeccione periódicas, una vez que ha superado una evaluación previa en la que se ha puesto de manifiesto su respeto de las disposiciones mínimas exigidas sobre seguridad marítima.

La implicación de la sociedad para la consecución de la eficiencia económica del Estado es aún si cabe más importante, ya que permite involucrar al ciudadano en la propia organización estatal, convirtiéndolo en sujeto básico de la misma; principio esencial de la propia configuración de Estado. Desde el momento en que el ciudadano valora como propios los logros y las deficiencias del sector público, actúa de forma responsable, colaborando en la consecución de los fines de bienestar, y no limitándose a actuar como un sujeto pasivo. Cuando el ciudadano adquiere la conciencia de partícipe del sector público, y observa los resultados positivos de su actuación, se convierte en garante de su eficiencia económica.

A\&C R. de Dir. Administrativo \& Constitucional, Belo Horizonte, ano 7, n. 30, p. 131-139, out./dez. 2007 
En el ámbito de la seguridad marítima, la implicación del ciudadano a través de actuaciones de voluntariado ha sorprendido a la propia Administración, que no ha desarrollado los medios adecuados para rentabilizar estas iniciativas sociales. Supuesto paradigmático de este movimiento ciudadano fue la participación de una marea de voluntarios de distintas nacionalidades, que acudieron masivamente a las costas españolas de Galicia para limpiar el vertido del buque Prestige; al igual que es necesario poner de manifiesto la destacada implicación ciudadana en la organización del Cuerpo de Guardacostas de Estados Unidos, que realiza una labor esencial para la protección de su entorno marítimo, y cuya labor ha merecido un destacado reconocimiento por parte de las autoridades competentes.

\section{Reflexiones finales}

Para finalizar, podemos afirmar que los modelos tradicionales de Estado se encuentran obsoletos, ya que se muestran incapaces de dar respuesta a las nuevas necesidades creadas por la sociedad, y el propio sector privado. La intervención del Estado del siglo XXI exige la cooperación con el sector privado, y la recuperación de la figura del ciudadano como instrumento clave. La eficiencia económica, y la eficacia en la prestación de los servicios público, y en cualquier prestación pública, objetivos básicos del Estado, son fines exigibles por el ciudadano y el sector privado. Manteniendo el nivel de gasto público es posible alcanzar los fines básicos del Estado, cuando el ciudadano adquiere la conciencia de buen hacer en la actuación de la Administración, colabora con la misma para alcanzar el nivel de eficacia exigido.

Por su parte, en lo que respecta a la relación con el sector privado, es imprescindible la implantación de dispociones estatales que hagan partícipe al sector privado no sólo en los beneficios, si no también en los costes directos de las actuaciones públicas. El equilibrio del mercado exige que todos los sujetos que participan en el mismo, tanto públicos como privados compartan costes y beneficios; la intervención de la Administración no puede verse reducida a un simple proveedor de subvenciones. El desafío de este nuevo modelo de Estado eficaz económicamente, es implicar al sector privado en la consecución de fines de interés social que, si bien en principio parecen no repercutir en sus ganancias, pueden ocasionar la ralentización económica de un Estado, con el subsiguiente perjuicio para

A\&C R. de Dir. Administrativo \& Constitucional, Belo Horizonte, ano 7, n. 30, p. 131-139, out./dez. 2007 
los intereses privados.

Las actuaciones propuestas no resultan extrañas a la realidad social actual, ya que son numerosos los ejemplos de voluntariado e implicación de los movimientos sociales en iniciativas de carácter social, educativo, ambiental, y de cualquier índole vinculada tradicionalmente a las actuaciones públicas. En el mismo sentido, las organizaciones profesionales y organismos de representación de sectores industriales y económicos de distinta naturaleza, ya están desarrollando iniciativas de interés público a través sus obras sociales y fundaciones, entre otros.

El sector público debe canalizar estos esfuerzos, para rentabilizarlos, y aprovechar su propia estructura organizativa para apoyar y fomentar estas iniciativas. El desarrollo de las mismas, también exige una conversión previa del propio entramado público, la simplificación y cercanía al ciudadano, así como la muestra constante de eficacia económica a nivel interno, lo cual pueda ser transmitido al ciudadano a través del principio de transparencia en sus actuaciones.

La eficiencia económica del Estado es una exigencia básica en las demandas ciudadanas; es un presupuesto que subyace a cualquier actuación pública y traspasa las fronteras entre lo público y lo privado, entre las decisiones administrativas y las decisiones de origen político. Si bien comenzamos este análisis manifestando las diferencias entre el sector público y el privado, debemos concluirlo poniendo de relieve el principio básico que deben compartir: la eficiencia económica, como medio para la obtención de mayores beneficios con una inversión de gastos suficiente y proporcionada.

Curitiba (Brasil), Mayo de 2007.

\section{Bibliografía}

CIAPPINA, C. Reflexiones en torno a Estado, Mercado y Tercer Sector. Documentos del Instituto Provincial de la Administración Pública, La Plata, 2004.

CORRALES, M. Modernización de los servicios públicos por redes: eficiencia económica y solidaridad social. Institución y Desarrollo, n. 3, 1999.

DURANT, R., FIORINO, D., O'LEARY, R. (Ed.). Environmental Governance Reconsidered. Challenges, Choices, and Opportunities. Massachusetts: The MIT Press, 2004.

GUDYNAS, E. Globalización, Políticas Sociales y Medio Ambiente. Revista del Centro de Estudios Latinoamericanos - TAREAS, Panamá, n. 98, p. 23-44, 1998.

KAST, M. Relaciones de la Política Económica con la Administración del Estado de Chile:

A\&C R. de Dir. Administrativo \& Constitucional, Belo Horizonte, ano 7, n. 30, p. 131-139, out./dez. 2007 
El Estado Empresario y el Principio de Subsidiariedad. Revista de Estudios Públicos, Centro de Estudios Públicos, n. 13, p. 211-228, 1984.

LECHNER, N. El debate sobre Estado y mercado. Nueva Sociedad, n. 121, p. 80-89.1992.

LECHNER, N. Las transformaciones de la política. Revista Mexicana de Sociología, v. 58, n. 1, p. 3-16, enero/marzo 1996.

LECHNER, N. ¿Cómo reconstruimos un nosotros? Boletín de la Revista Latinoamericana de Desarrollo Humano, Programa de las Naciones Unidas para el Desarrollo (PNUD), n. 15, nov. 2005.

MARTÍNEZ, N. H. Estado de Derecho y Eficiencia Económica. In: CARRILLO, F.; JARQUÍN, E. (Ed.). La Economía Política de la Reforma Judicial. Washington: Banco Interamericano de Desarrollo, 1997.

RODRÍGUEZ-ARANA MUÑOZ, J. Ética institucional: Mercado versus Función Pública. Madrid: Dykinson, 1996.

RODRÍGUEZ-ARANA MUÑOZ, J. Nuevas claves del Estado del Bienestar. Hacia la Sociedad del Bienestar. Granada: Comares, 1998.

SÁNCHEZ BLANCO, A. El sistema económico de la Constitución Española: participación institucional de las autonomías territoriales y dinámica social en la economía. Madrid: Civitas, 1992.

VIG, N., FAURE, M., (Ed.). Green Giants? Environmental Policies of the United States and the European Union. Massachusetts: The MIT Press, 2004.

Informação bibliográfica deste texto, conforme a NBR 6023:2002 da Associação Brasileira de Normas Técnicas (ABNT):

ZAMORA ROSELLÓ, María Remedios. ¿Eficiencia económica del Estado como substituto a la ética pública convencional? Desafíos actuales del Estado para la consecución de la eficiencia económica. Su aplicación en el sector de la seguridad marítima. A\&C Revista de Direito Administrativo \& Constitucional, Belo Horizonte, ano 7, n. 30, p. 131-139, out./dez. 2007. 